Proceedings of the International Symposium on Physics of Materials (ISPMA 14), September 10-15, 2017, Prague

\title{
Spin-Wave Spectroscopy and Magnetostructural Studies of Co Nanogranular Composites
}

\author{
E.A. Denisova ${ }^{a, b, *}$, S.V. Komogortsev ${ }^{a}$, R.S. Iskhakov ${ }^{a}$, L.A. Chekanova ${ }^{a}$, \\ YU.E. KALININ ${ }^{c}$ AND A.V. SitniKOV ${ }^{c}$ \\ ${ }^{a}$ Institute of Physics, Federal Research Center KSC SB RAS, \\ Krasnoyarsk, Akademgorodok 50, bld. 38, Russian Federation \\ ${ }^{b}$ Siberian Federal University, Krasnoyarsk, Russian Federation \\ ${ }^{c}$ Voronezh State Technical University, Voronezh, Russian Federation
}

\begin{abstract}
Nanogranular $\mathrm{Co}-\mathrm{SiO}_{2}$, and $\mathrm{Co}-\mathrm{CaF}_{2}$ films are investigated using ferromagnetic resonance and magnetic measurements. The spectrum of standing spin-wave has been registered in the perpendicular experiment configuration for all types of films with the magnetic granules content above 52 vol.\%. The dependences of resonance fields $H_{r}$ of spin modes on the mode number squared $H_{r}\left(n^{2}\right)$ are non-linear. Such behavior is supposed to be the result of the fluctuations of magnetic parameters (the magnetization and the ferromagnetic exchange coupling) in nanocomposite films. A comparison between the magnetic properties of $\mathrm{Co}-\mathrm{SiO}_{2}$ granular films and $\mathrm{Co}-\mathrm{CaF}{ }_{2}$ films is carried out. The surface anisotropy value $K_{s}$ of $\mathrm{Co}-\mathrm{SiO}_{2}$ films is evaluated from the surface resonance modes of spin-wave resonance spectra as $3.5 \times 10^{-4}<K_{s}<7 \times 10^{-4} \mathrm{~J} / \mathrm{m}^{2}$. The magnetic anisotropy of $\mathrm{Co}_{100-x}\left(\mathrm{SiO}_{2}\right)_{x}$, $\mathrm{Co}_{100-x}\left(\mathrm{CaF}_{2}\right)_{x}$ films $(34<x<74)$ is investigated. The surface magnetic anisotropy of granules $k_{s}$ and the surface magnetic anisotropy of films $K_{s}$ are determined. It is found that surface anisotropy of granules is predominant.
\end{abstract}

DOI: 10.12693/APhysPolA.134.623

PACS/topics: $76.50 .+\mathrm{g}, 75.30 . \mathrm{Gw}$

\section{Introduction}

Granular magnetic films with the soft magnetic granules and nonmagnetic matrix with low conductivity are promising magnetic nanostructures for high-frequency applications due to their high resistivity and a high magnetic permeability [1-5]. The unique nanostructure and relative simplicity of changing the particle size and concentration in granular materials make these systems almost ideal for investigations of the physical properties of nanostructured systems and approbation of novel techniques of nanostructural materials investigation [6-8]. Analysis of the ferromagnetic resonance (FMR) and spinwave resonance (SWR) spectra combined with magnetometric investigations allows obtaining information on the exchange coupling and its fluctuations in inhomogeneous media [9]. The saturation magnetization $M_{s}$ and magnetic anisotropy constant can be determined using measurements of uniform modes of ferromagnetic resonance [10]. The observation of surface mode can be used to determine the surface anisotropy constant $K_{s}$ [11]. Spin-wave resonance enables us to study the energy spectra of exchange spin waves in thin ferromagnetic films directly through experiments [12]. The dispersion law for spin waves contains information about inhomogeneities of the fundamental magnetic constants [9]. Fluctuations of the different parameters of the spin system lead to qualitatively different types of the dispersion law modification $[9,13-15]$.

*corresponding author; e-mail: len-den@iph.krasn.ru
It is known that the magnetic properties of nanostructured ferromagnets critically depend on their micromagnetic structure. If exchange interaction between nanoparticles of the composite is strong, relations of the macroscopic magnetic parameters (such as coercivity, magnetic susceptibility) and microscopic parameters of the spin system, such as particles size (grains $R_{c}$ ), inter-grain exchange interaction, local anisotropy, are described by the random anisotropy model $[16,17]$. The random anisotropy destroys long-range ferromagnetic order in the spin system, but it is preserved on the scale of magnetic orientation coherence $R_{L}$ due to exchange interaction. It was shown that microscopic magnetic properties of nanomagnets are governed by size $2 R_{L}$ and anisotropy of stochastic domains that are formed spontaneously by large number of particles (of size $2 R_{c}$ ). The correlation magnetometry technique allows us to derive size of the element of micromagnetic structure of nanomagnet (stochastic domain), magnitude of effective anisotropy in the region and size of elements of the nanostructure (size of nanoparticles) and its local anisotropy, and spatial dimensionality of the system of exchange-coupled particles from the curves of magnetization approaching saturation [17]. In this paper, we use grain size dependence of local magnetic anisotropy to separate volume and surface contributions to anisotropy.

The aim of this work is to investigate the magnetic properties and magnetic microstructure of $\mathrm{Co}-\mathrm{SiO}_{2}$ and $\mathrm{Co}-\mathrm{CaF}_{2}$ granular films by correlation magnetometry and SWR spectroscopy techniques. In the paper we will demonstrate that the magnetostructure techniques allow us to obtain additional information on microstructure of composite material. 


\section{Experiment}

Nanogranular films with a nominal composition of $\mathrm{Co}_{100-x}\left(\mathrm{SiO}_{2}\right)_{x}$ and $\mathrm{Co}_{100-x}\left(\mathrm{CaF}_{2}\right)_{x}(34<x<74)$ were produced by the ion-beam sputtering. The details on the films fabrication, the particle size distribution, and the microstructure of the composite films were reported elsewhere [18, 19]. The transmission electron microscopy (TEM) photographs of the $\mathrm{Co}-\mathrm{SiO}_{2}$ and $\mathrm{Co}-\mathrm{CaF}_{2}$ granular films show a system of particles with sizes varying from $3-4$ to $6-8 \mathrm{~nm}$ when $x$ increases from 34 to $74 \%$. In the $\mathrm{Co}-\mathrm{SiO}_{2}$ system, the investigated samples do not have well insulated metal particles, due to the Co particles agglomerates forming. From the nuclear magnetic resonance and electron diffraction results it can be concluded that the metal granules were characterized by the nearest environment of Co atoms with predominantly hcp structure in the whole concentration range. The $\mathrm{X}$-band FMR spectra at $9.2 \mathrm{GHz}$ were measured at room temperature using a standard EPR spectrometer. The magnetization curves were measured using vibrating sample magnetometer in the fields of up to $1100 \mathrm{kA} / \mathrm{m}$ applied in the film plane. Information on local anisotropy field was obtained from approach to saturation magnetization law.

\section{Results and discussion}

Temperature dependence of magnetization in films is well fitted by the Bloch $T^{3 / 2}$ law. The value of $B$ (the Bloch constant) decreases when ferromagnetic phase concentration increases from $40 \times 10^{-6} \mathrm{~K}^{-3 / 2}$ to $3 \times 10^{-6} \mathrm{~K}^{-3 / 2}$ for $\mathrm{Co}-\mathrm{SiO}_{2}$ and $\mathrm{Co}-\mathrm{CaF}_{2}$ films. The exchange interaction constant $A$ was calculated using standard expression

$$
A_{\text {eff }}=\frac{k_{\mathrm{B}}}{8 \pi}\left(\frac{M_{0}}{g \mu_{\mathrm{B}}}\right)^{1 / 3}\left(\frac{2.612}{B}\right)^{2 / 3}
$$

where $k_{\mathrm{B}}$ is the Boltzmann constant, $g=2, \mu_{\mathrm{B}}$ is the Bohr magneton, $M_{0}$ is the saturation magnetization at $0 \mathrm{~K}$. The $A$ magnitude increases when ferromagnetic phase concentration increases from $3.6 \times 10^{-12}$ to $1.68 \times 10^{-11} \mathrm{~J} / \mathrm{m}$ for $\mathrm{Co}-\mathrm{SiO}_{2}$ films and from $3 \times 10^{-12}$ to $2 \times 10^{-11} \mathrm{~J} / \mathrm{m}$ for $\mathrm{Co}-\mathrm{CaF}_{2}$ films.

Ferromagnetic resonance spectra of the $\mathrm{Co}-\mathrm{SiO}_{2}$ and $\mathrm{Co}-\mathrm{CaF}_{2}$ nanogranular films are studied for various orientations of the film plane with respect to the external field. The FMR spectrum for magnetic field $(H)$ applied parallel to the film plane consists of a single absorption line for all types of samples. The FMR linewidth $\Delta H$ decreases with increasing ferromagnetic phase content when magnetic field is applied in the film plane, reflecting the magnetic inhomogeneity of the film with low ferromagnetic phase content. The separation of granules in a nonmagnetic matrix should decrease the dipole-dipole interaction between the granules and thus should cause narrowing of the resonance line $[6,20,21]$. However, TEM shows that the Co particles form agglomerates with a wide distribution of particle shape and size, leading to a corresponding wide distribution of resonance fields and line widths.
Figure 1 shows the dependences of resonance fields on volume fraction of magnetic phase for the $\mathrm{Co}-\mathrm{SiO}_{2}$ and $\mathrm{Co}-\mathrm{CaF}_{2}$ nanogranular films. Extrapolations of transverse and longitudinal $H_{r}(X)$ dependences to the low $x$ values reveal an intersection at $H_{r}=\omega / \gamma=$ $2.4 \times 10^{5} \mathrm{~A} / \mathrm{m}$, that corresponds to a resonance in isolated spherical granules. The volume fraction of magnetic granules at the point of convergence is $x_{i} \approx 30 \%$ for $\mathrm{Co}-$ $\mathrm{SiO}_{2}$ and $x_{i} \approx 35 \%$ for $\mathrm{Co}-\mathrm{CaF}_{2}$. The convergence point, $x_{i}$, should be distinguished from the percolation threshold, $x_{C}$. At the point $x=x_{i}$ the fraction of superparamagnetic granules becomes close to $100 \%$, and the fraction of ferromagnetic granules becomes negligible. As a result, the macroscopic magnetic anisotropy disappears. The FMR linewidth of $\mathrm{Co}-\mathrm{CaF}_{2}$ films is less than those of $\mathrm{Co}-\mathrm{SiO}_{2}$ films. When the external field is applied perpendicular to the film plane it is possible to observe multipeaked spectra, due to resonance absorption by standing spin-wave resonance modes. There are up to 6 peaks in $\mathrm{SWR}$ spectrum of the $\mathrm{Co}-\mathrm{SiO}_{2}$ films with $x>52 \%$ ferromagnetic phase. The SW modes critically depend on some form of magnetic inhomogeneities e.g., on the presence of a magnetization gradient or other magnetic variation through the film. Such nonuniformities can either exist at the surfaces of the film playing a pivotal role in determining surface anisotropy and spin pinning conditions, alternatively they can exist throughout the bulk of the film. The boundary conditions for the standing spin-wave (surface spin pinning conditions) in this layer are formed during sputtering process of composite films.

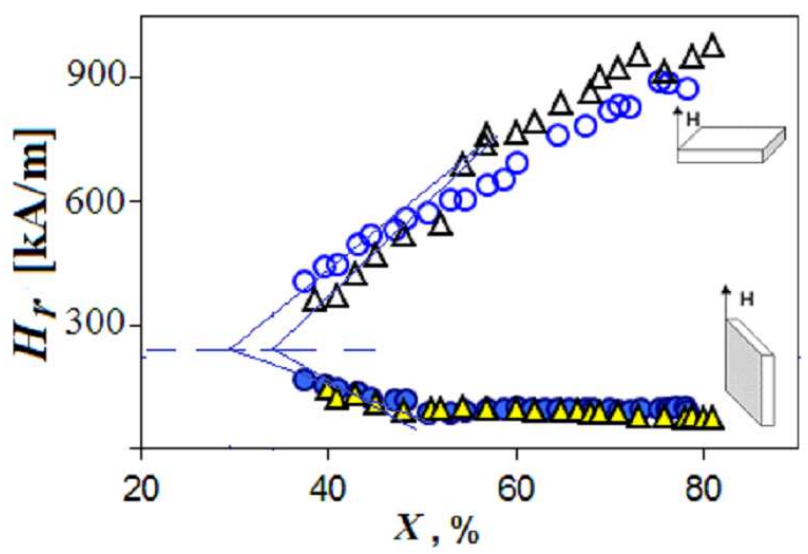

Fig. 1. The dependences of resonance fields for $\mathrm{Co}^{-}$ $\mathrm{SiO}_{2}$ (circle) and $\mathrm{Co}-\mathrm{CaF}_{2}$ (triangle) granular films on volume of magnetic phase (open markers - the external field is applied perpendicular to the film plane, filled markers - the external field is applied parallel to the film plane).

Typical SWR spectrum of $\mathrm{Co}-\mathrm{SiO}_{2}$ films is presented in Fig. 2. It is found that the $H_{r}\left(n^{2}\right)$ (where $n$ is the mode number) dependences for both types of the granular films are non-linear (Fig. 2b). The modification of $H_{r}\left(n^{2}\right)$ dependence like shown in Fig. $2 \mathrm{~b}$ indicates the predomi- 
nance of exchange coupling heterogeneities [9, 13-15]. A surface resonance mode is observed for $\mathrm{Co}-\mathrm{SiO}_{2}$ films. It means that there was the easy-plane anisotropy in these films. The surface anisotropy value is evaluated using the equation [11]:

$$
K_{s}=\sqrt{\frac{\left(H_{s}-H_{1}\right) A M_{\mathrm{eff}}}{2}},
$$

where $H_{s}$ is the resonance field that corresponds to the surface mode. The surface anisotropy value $K_{s}$ of $\mathrm{Co}-\mathrm{SiO}_{2}$ films was evaluated from the surface resonance modes of SWR spectra as $3.5 \times 10^{-4}<K_{s}<$ $7 \times 10^{-4} \mathrm{~J} / \mathrm{m}^{2}$.

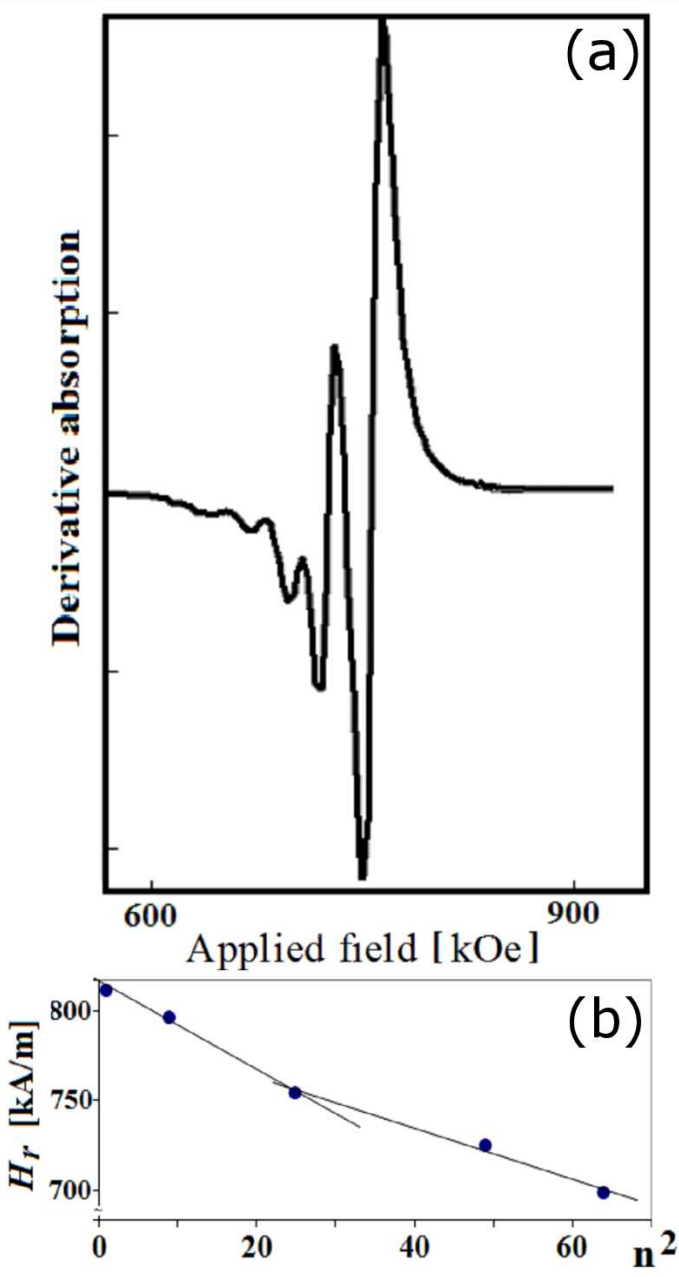

Fig. 2. Spin-wave resonance spectrum of $\mathrm{Co}_{55}\left(\mathrm{CaF}_{2}\right)_{45}$ granular film (a) and corresponding dependence of resonance fields $H_{r}$ of spin modes on the mode number squared (b).

There is no magnetic anisotropy in the film plane according to magnetization curve studies for all the samples. The magnetization curves $M(H)$ for granular films at $300 \mathrm{~K}$ are well fitted by the expression [21]:

$$
M(H)=M_{s}\left(1-\frac{H_{a}^{2} H^{-1 / 2}}{15\left(H^{3 / 2}+H_{R}^{3 / 2}\right)}\right),
$$

where $H_{a}=2 K_{g} / M_{s}$ is the local magnetic anisotropy field (magnetic anisotropy of granule) and $H_{R}=$ $2 A / M_{s} R_{c}^{2}$ is exchange field, where $A$ is the exchange stiffness, $M_{s}$ is magnetization and $R_{c}$ is the granule size. Equation (3) is derived for the random anisotropy model. The macroscopic anisotropy field in a stochastic domain is defined as $\left\langle H_{a}\right\rangle_{L}=2 K_{L} / M_{s}=2 A / M_{s} R_{L}^{2}$ and can be estimated for given values of $H_{a}$ and $H_{R}$ according to $\left\langle H_{a}\right\rangle_{L}=H_{a}^{4} / H_{R}^{4}$. The values of local magnetic anisotropy field $H_{a}$ and anisotropy field of stochastic magnetic domain $\left\langle H_{a}\right\rangle_{L}$ are found to decrease with increase of volume fraction of the magnetic phase. The magnetic anisotropy energy constant of granule, $K_{g}=H_{a} M_{s} / 2$, and anisotropy energy constant of stochastic magnetic domain, $K_{L}=\left\langle H_{a}\right\rangle_{L} M_{s} / 2$, are calculated using fitted parameters $H_{a}$ and $H_{R}$.
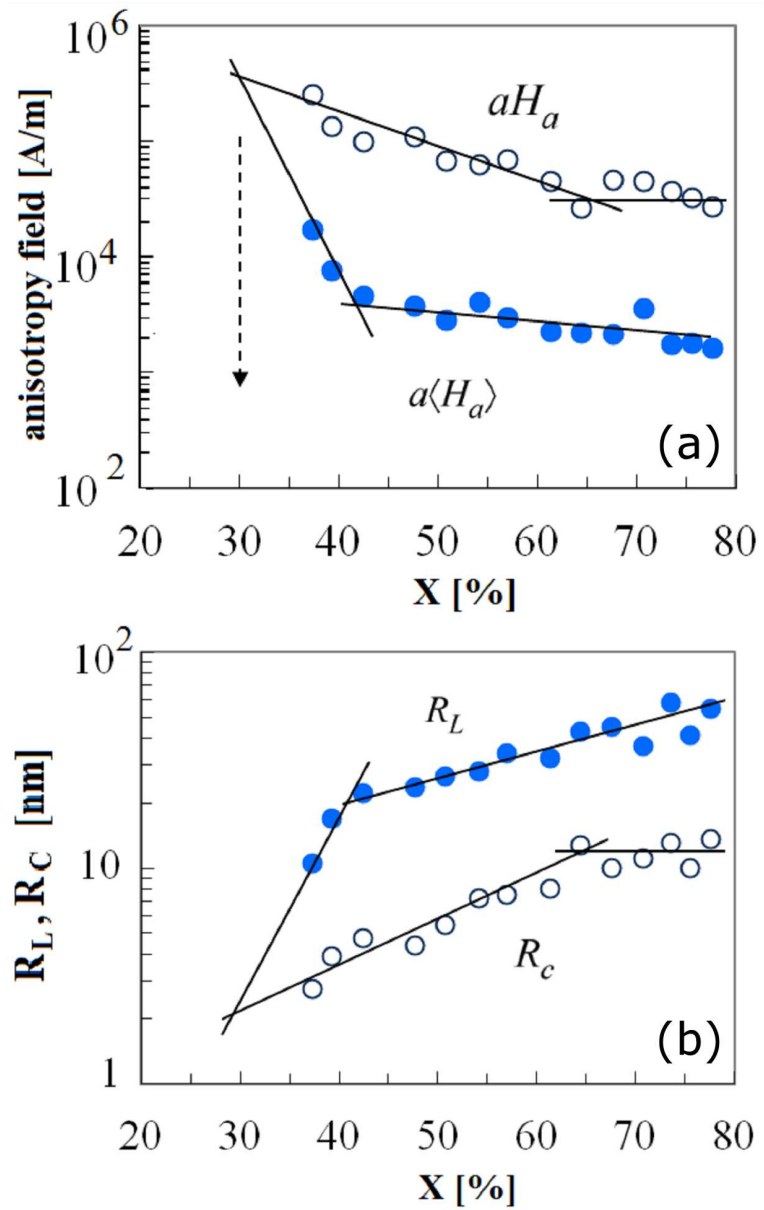

Fig. 3. The local magnetic anisotropy field $a H_{a}$ and the magnetic anisotropy field of stochastic magnetic domain $a\left\langle H_{a}\right\rangle$ for $\mathrm{Co}_{x}\left(\mathrm{SiO}_{2}\right)_{1-x}$ films as a function of the magnetic phase fracture (a). The size of stochastic magnetic domain $R_{L}$ and structural correlation length $R_{C}$ in the composite films $\mathrm{Co}_{x}\left(\mathrm{SiO}_{2}\right)_{1-x}$.

Resulting values of $\left\langle H_{a}\right\rangle_{L}$ and $H_{a}$ are shown in Fig. 3 for different granule concentrations, $x$. The extrapolation of these dependences to the low $x$ allows us to estimate the percolation threshold by interparticle exchange as a 
point of convergence $X_{C} \approx 30 \%$. Using definition of exchange field $H_{R}$ and obtained values of $M_{s}$, and $A$, we estimate $R_{c}$ and $R_{L}$ for granular films. The dependences of $R_{c}$ and $R_{L}$ on metal phase content are shown in Fig. $3 \mathrm{~b}$. It is seen that the linear parts of $R_{c}(x)$ and $R_{L}(x)$ dependences intersect at the same point as $\left\langle H_{a}\right\rangle_{L}(x)$ and $H_{a}(x)$ dependences.

Figure 4 shows that magnetic anisotropy of granules $K_{g}$ is a decreasing function of granule size $D$. The following equation that takes into account both, surface and volume anisotropies to the local anisotropy energy of magnetic granule is used to fit the data [22-25]:

$$
K_{g}=K_{V}+6 k_{S} / D
$$

The resulting values are found to be $k_{s}=3.4 \times 10^{-3} \mathrm{~J} / \mathrm{m}^{2}$ and $K_{V} \approx 4 \times 10^{4} \mathrm{~J} / \mathrm{m}^{3}$ for $\mathrm{Co}-\mathrm{SiO}_{2}$ films and $k_{s}=$ $2.8 \times 10^{-3} \mathrm{~J} / \mathrm{m}^{2}$ and $K_{V} \approx 10^{4} \mathrm{~J} / \mathrm{m}^{3}$ for $\mathrm{Co}-\mathrm{CaF}_{2}$ films. It was found that surface anisotropy of granules is predominant.

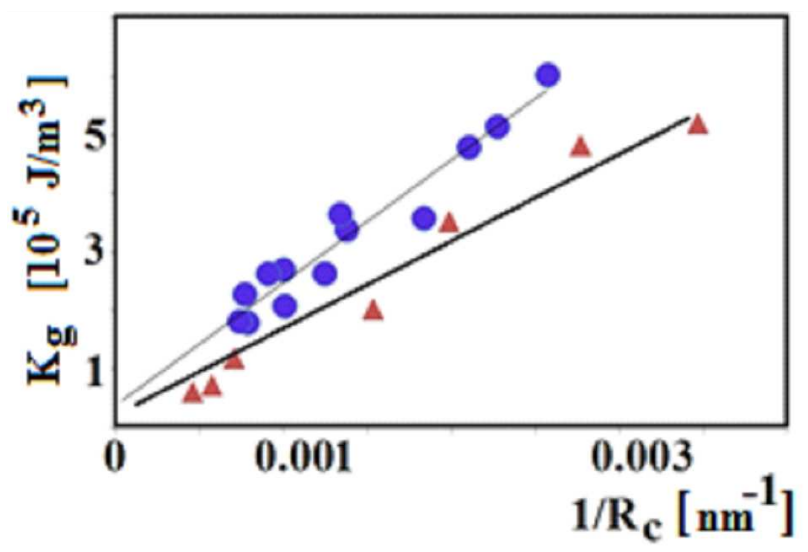

Fig. 4. The size dependence of anisotropy constant of individual granules $K_{g}$ for $\mathrm{Co}-\mathrm{SiO}_{2}$ (circle) and $\mathrm{Co}-$ $\mathrm{CaF}_{2}$ (triangle) granular films. The solid line - equation $K_{g}=K_{V}+6 k_{g} / D$

\section{Conclusions}

The magnetic microstructure and spin-wave resonance features of $\mathrm{Co}-\mathrm{SiO}_{2}$ and $\mathrm{Co}-\mathrm{CaF}_{2}$ films $(34<x<74)$ produced by ion-beam sputtering were investigated. The spectrum of standing spin-wave has been detected in the perpendicular experiment configuration for films with the magnetic phase content above 52 vol.\%. It is found that the FMR linewidth of $\mathrm{Co}-\mathrm{CaF}_{2}$ nanocomposites is much smaller than for nanocomposites with disorder $\mathrm{SiO}_{2}$ matrix. The type of the dispersion relation of spin waves in $\mathrm{Co}-\mathrm{SiO}_{2}$ and $\mathrm{Co}-\mathrm{CaF}_{2}$ composite films is affected by the exchange coupling fluctuations. The surface anisotropy value of $\mathrm{Co}-\mathrm{SiO}_{2}$ and $\mathrm{Co}-\mathrm{CaF}_{2}$ films was evaluated from the surface resonance modes of SWR spectra as $3.5 \times 10^{-4}<K_{s}<7 \times 10^{-4} \mathrm{~J} / \mathrm{m}^{2}$. Information on local anisotropy field was obtained from investigation of approach to saturation magnetization law. It was found that surface anisotropy of granules $k_{s}$ is predominant $\left(k_{s}=3.4 \times 10^{-3} \mathrm{~J} / \mathrm{m}^{2}\right.$ for $\mathrm{Co}-\mathrm{SiO}_{2}$ films and $k_{s}=2.8 \times 10^{-3} \mathrm{~J} / \mathrm{m}^{2}$ for $\mathrm{Co}^{-} \mathrm{CaF}_{2}$ films).

\section{Acknowledgments}

This work was supported by RFBR grants nos. 16-0300256, 16-03-00969.

\section{References}

[1] C.L. Chien, J. Appl. Phys. 69, 5267 (1991).

[2] Y.D. Zhan, S.H. Wang, D.T. Xiao, J.I. Budnick, W.A. Hines, IEEE Trans. Magn. 37, 2275 (2001).

[3] K.D. Coonley, G.J. Mehas, C.R. Sullivan, U.J. Gibson, IEEE Trans. Magn. 36, 3463 (2000).

[4] T.N. Koltunowicz, P. Zhukowski, V. Bondariev, A. Saad, J.A. Fedotova, A.K. Fedotov, M. Milosavljević, J.V. Kasiuk, J. Alloys Comp. 615, S361 (2014).

[5] S.V. Komogortsev, E.A. Denisova, R.S. Iskhakov, A.D. Balaev, L.A. Chekanova, Y.E. Kalinin, A.V. Sitnikov, J. Appl. Phys. 113, 17C105 (2013).

[6] G.N. Kakazei, A.F. Kravets, N.A. Lesnik, M.M. Pereira de Azevedo, Y.G. Pogorelov, J.B. Sousa, J. Appl. Phys. 85, 5654 (1999).

[7] H.S.M. Al'Azzavi, A.B. Granovskii, Y.E. Kalinin, V.A. Makagonov, A.V. Sitnikov, O.S. Tarasova, Phys. Solid State 58, 938 (2016).

[8] A. Butera, T.J. Klemmer, J.A. Barnard, J. Appl. Phys. 83, 4855 (1998).

[9] V.A. Ignatchenko, R.S. Iskhakov, JETP 48, 726 (1978).

[10] C. Kittel, Phys. Rev. 73, 155 (1948).

[11] J. Gómez, A. Butera, J.A. Barnard, Phys. Rev. B 70 , 54428 (2004).

[12] M.H. Seavey, P.E. Tannenwald, Phys. Rev. Lett. 1, 168 (1958).

[13] V.A. Ignatchenko, R.S. Iskhakov, L.A. Chekanova, N.S. Chistyakov, JETP 48, 328 (1978).

[14] L.J. Maksymowicz, D. Sendorek-Temple, R. Żuberek, J. Magn. Magn. Mater. 58, 303 (1986).

[15] R.S. Iskhakov, M.M. Brushtunov, A.S. Chekanov, Fiz. Tverd. Tela 29, 2699 (1987)

[16] G. Herzer, Acta Mater. 61, 718 (2013).

[17] R.S. Iskhakov, S.V. Komogortsev, Phys. Met. Metallogr. 112, 666 (2011).

[18] O.V. Stognei, A.V. Sitnikov, Phys. Solid State 52 , 2518 (2010)

[19] Y.E. Kalinin, A.V. Sitnikov, O.V. Stognei, I.V. Zolotukhin, P.V. Neretin, Mater. Sci. Eng. A 304-306, 941 (2001).

[20] R.S. Iskhakov, E.A. Denisova, S.V. Komogortsev, L.A. Chekanova, Y.E. Kalinin, A.V. Sitnikov, Phys. Solid State 52, 2263 (2010).

[21] S.V. Komogortsev, R.S. Iskhakov, J. Magn. Magn. Mater. 440, 213 (2017).

[22] Y. Labaye, O. Crisan, L. Berger, J.M. Greneche, J.M.D. Coey, J. Appl. Phys. 91, 8715 (2002).

[23] D.A. Dimitrov, G.M. Wysin, Phys. Rev. B 50, 3077 (1994).

[24] A. Millan, A. Urtizberea, N.J.O. Silva, F. Palacio, V.S. Amaral, E. Snoeck, V. Serin, J. Magn. Magn. Mater. 312, L5 (2007).

[25] J. Restrepo, Y. Labaye, J.M. Greneche, Physica B Condens. Matter 384, 221 (2006). 